\title{
Relação entre a densidade básica e as retrações em madeira de teca
}

\author{
Ana Carolina Caixeta Dias ${ }^{1}$, Raquel Marchesan ${ }^{2 *}$, Vanessa Coelho Almeida ${ }^{2}$, Thiago Campos \\ Monteiro $^{3}$, Cristiano Bueno de Moraes ${ }^{2}$
}

\author{
${ }^{1}$ Programa de Pós-Graduação em Ciências Florestais e Ambientais, Universidade Federal do Tocantins, Gurupi - TO, Brasil. \\ ${ }^{2}$ Departamento de Engenharia Florestal, Universidade Federal do Tocantins, Gurupi - TO, Brasil. \\ ${ }^{3}$ Departamento de Engenharia Florestal, Universidade Federal de Minas Gerais, Montes Claros - MG, Brasil.
}

\begin{abstract}
RESUMO O estudo objetivou determinar a densidade básica, o colapso, a retração, e o coeficiente de anisotropia da madeira de teca e estudar a associação entre as referidas propriedades físicas, visando definir sua qualidade. Foram utilizadas madeiras provenientes do estado de Minas Gerais com dimensões de 20 × 20 x 50 mm nas direções radial, tangencial e longitudinal, respectivamente. As determinações da densidade básica e das retrações foram realizadas de acordo com a NBR 11941 e NBR 7190 respectivamente. O colapso foi calculado pela deformação da área inicial pela área final. O processamento dos dados foi realizado através da análise estatística descritiva, da análise de trilha e componentes principais. Os resultados apresentaram valores de densidade básica média das amostras de $0,510 \mathrm{~g} \cdot \mathrm{cm}^{-3}$ e colapso moderado $(2,53 \mathrm{~mm})$. A retração volumétrica parcial $(3,6 \%)$ e total $(5,23 \%)$ apresentaram comportamento estável, justificando o baixo valor de coeficiente de anisotropia (1,99\%), indicando satisfatória estabilidade dimensional. A análise de correlação não apresentou significância entre a densidade com demais parâmetros. Na análise dos componentes principais (PC), apresentou maior correlação entre as retrações totais no PC1 e as retrações parciais e o colapso no PC2. Portanto, para a madeira de teca não houve relação entre a densidade básica e as demais propriedades avaliadas.

Palavras-chave: Análise de trilha; Colapso; Retratibilidade; Tectona grandis.
\end{abstract}

\section{Relationship between basic density and shrinkage in teca wood}

\begin{abstract}
The objective of this study was to determinate the basic density, collapse, retraction, and anisotropy coefficient of teca wood and to study the association between these physical properties, aiming to define it's quality. The timber used was from the state of Minas Gerais, with dimensions of $20 \times 20 \times 50 \mathrm{~mm}$ in the radial, tangential and longitudinal directions, respectively. The determinations of the basic density and retractions were performed according to the NBR 11941 and NBR 7190 respectively. The collapse was calculated by the deformation of the initial area by the final area. The data processing was performed by descriptive statistical analysis, the path analysis and principal components. The results showed average basic density values of the samples from $0.510 \mathrm{~g} . \mathrm{cm}^{-3}$ and moderate collapse $(2.53 \mathrm{~mm})$. The partial volumetric retraction (3.6\%) and total (5.23\%) presented a stable behavior, justifying the low value of anisotropy coefficient (1.99\%), indicating dimensional stability. The correlation analysis did not present significance between density and other parameters. In the analysis of the main components (PC), there was a greater correlation between total retractions in PC1 and partial retractions and collapse in PC2. Therefore, the teak wood didn't have any relation between the basic density and other evaluated properties.
\end{abstract}

Keywords: Path analysis; Collapse; Retractivity; Tectona grandis.

\section{Introdução}

Devido às suas características tecnológicas como a elevada estabilidade dimensional, média densidade $\left(0,55 \mathrm{~g} / \mathrm{cm}^{3}\right)$, durabilidade natural, trabalhabilidade e coloração chamativa, a madeira de teca adquiriu reconhecimento mundial com emprego em produtos de finalidades decorativas, construções navais, construção civil de fins nobres, assoalhos e decks, setor mobiliário, indústria de laminados e adornos em geral (MOTTA, 2013; IBÁ, 2014). Diante disto, o crescimento do uso da madeira de teca na indústria de base florestal tem dado destaque a este produto na economia nacional, aumentando 
a necessidade de estudos voltados para a qualidade da madeira.

Os primeiros maciços florestais de teca em escala comercial iniciaram-se no estado de Mato Grosso, no município de Cárceres, no final da década de 60 e início da década de 70, por iniciativa da Empresa Cáceres Florestal S.A. Segundo a Indústria Brasileira de Árvores - IBÁ (IBA, 2015), no Brasil a estimativa da área plantada de teca foi de 87.499 ha no ano de 2014, distribuídos entre os estados do Acre, Amapá, Mato Grosso do Sul, Paraná, Rondônia, São Paulo, Tocantins e, principalmente, Mato Grosso e Pará.

A densidade básica é considerada uma das principais propriedades físicas da madeira por ser uma variável de fácil determinação e uma forte indicadora de qualidade da madeira. Na prática, serve como uma referência para a classificação das madeiras (GONÇALVES et al., 2009). Essa propriedade possui relação direta com diversos parâmetros, dentre eles, a retratibilidade, trabalhabilidade, durabilidade natural e proprie dades mecânicas. Além disso, pode influenciar no aparecimento de defeitos como rachaduras e colapso (KLITZKE; BATISTA, 2010; SILVEIRA et al., 2013).

Para Klitzke (2003) o colapso acontece quando os esforços da tensão capilar ultrapassam a resistência à compressão perpendicular à grã da parede celular a qual ocorre quando os meniscos se movem através das pontuações da parede celular. Essa reação provoca tensões capilares nas células resultando em deformações na superfície da peça de madeira podendo inviabilizar totalmente seu uso (SKAAR, 1972).

Assim como a densidade, a retratibilidade é considerada uma propriedade física importante, podendo afetar e limitar consideravelmente o emprego de espécies de madeiras em determinados produtos. Devido ao caráter anisotrópico da madeira, as variações dimensionais ocorrem distintamente na direção radial, tangencial e longitudinal. Sendo assim, torna- se necessário determinar a retratibilidade volumétrica total e as variações dimensionais lineares.

Outro índice utilizado é o coeficiente de anisotropia, obtido pela relação entre a retração tangencial e a radial. Segundo Gonçalves et al. (2009) este parâmetro é importante, pois prevê o comportamento da madeira em relação à secagem, podendo indicar maior ou menor propensão a defeitos de empenamentos, rachaduras e colapso.

Sendo assim, o objetivo deste estudo foi determinar a densidade básica, o colapso, a retração e o coeficiente de anisotropia da madeira. Ainda, objetivou-se realizar análises que demonstram associação entre as variáveis, visando definir a qualidade de sua madeira oriunda de um plantio experimental do estado de Minas Gerais.

\section{Material e Métodos}

\section{Matéria prima e confecção dos corpos de prova}

O material utilizado neste estudo foi oriundo de um plantio florestal com 13 anos de idade, localizado no município de Vazante, estado de Minas Gerais. Foram confeccionados corpos de prova com dimensões lineares de $20 \times 20 \times 50 \mathrm{~mm}$ a partir de discos obtidos da base ao topo das árvores, de acordo com a Associação Brasileira de Normas Técnicas - ABNT (1997) NBR 7190. Os corpos de prova passaram por um processo de lixamento manual para a eliminação de imperfeições e classificação verificando-se a existência ou não de defeitos (brocas, rachaduras, encurvamento) e a correta orientação nos sentidos radial, tangencial e longitudinal.

Os corpos de prova foram imersos em água e submetidos ao vácuo até a completa saturação. Posteriormente foram encaminhados para a estufa à temperatura constante (103 \pm $2^{\circ} \mathrm{C}$ ), pesados e medidos de $1 \mathrm{em} 1$ hora até atingirem peso 
constante. Este processo foi realizado para obtenção das dimensões no ponto de saturação das fibras (PSF), na umidade de equilíbrio (UE) e na condição seca ( $0 \%$ de umidade).

\section{Propriedades físicas}

A determinação da densidade básica foi realizada de acordo com a norma NBR 11941 (ABNT, 2003) pelo método da balança hidrostática. Utilizando-se os mesmos corpos de prova do ensaio da densidade básica, foram determinadas as porcentagens de colapso, retração da madeira e coeficiente de anisotropia.

A determinação do colapso foi calculada seguindo a metodologia utilizada por Welling, (1994). Após a coleta das deformações o colapso foi calculado por meio da Equação 1.

$C=\alpha i-\alpha f$

Em que: $C=$ Colapso $(\%) ; \alpha \mathrm{i}=$ área inicial $(\mathrm{mm}) ; \alpha \mathrm{f}_{=}$área final deformada (mm).

A retração da madeira foi determinada por meio da norma NBR 11941 (2003), onde foram medidas as dimensões lineares nos sentidos radial, tangencial e longitudinal e calculadas as retrações volumétricas parciais e totais para todos os corpos de prova. Os corpos de prova medidos foram saturados em água e secos em estufa até o peso constante. Para a retração volumétrica parcial, obteve-se a umidade dos corpos de prova no PSF e na UE (Equação 2). Este parâmetro foi avaliado partindo do pressuposto que na UE tem-se a real retração da madeira, sendo que as madeiras são utilizadas nestas condições de umidade. Para retração volumétrica total as amostras foram medidas no PSF e secas em estufa até atingir peso constante (0\%) (Equação 3).

$ß_{P}=\frac{(D . P S F-D \cdot U E)}{D . P S F} x 100$

$ß_{T}=\frac{(D . P S F \quad)}{D . P S F} x 100$

Em que: $\aleph_{\mathrm{P}}$ : retração máxima parcial, em uma dada direção estrutural (\%); $\beta_{\mathrm{T}=}$ retração máxima total, em uma dada direção estrutural (\%); $D_{P S F}=$ dimensão no ponto de saturação das fibras, em uma dada direção estrutural (mm); $D_{U E}=$ dimensão na umidade de equilíbrio, em uma dada direção estrutural $(\mathrm{mm}) ; \mathrm{D}_{\mathrm{S}=}$ dimensão absolutamente seca, em uma dada direção estrutural (mm).

O coeficiente de anisotropia foi obtido a partir da relação das direções tangenciais e radiais (Equação 4).

$A=\frac{\beta \mathrm{t}}{\beta r}$

Em que: $A=$ coeficiente de anisotropia (adimensional); $ß \mathrm{t}$ = Retração tangencial \%; $ß r$ = Retração radial \%.

\section{Análise estatística dos dados}

Os dados foram processados por meio de análise estatística descritiva com os valores obtidos nas medições dos planos fundamentais, ou seja, direções radiais e tangenciais. Obteve-se a média para cada parâmetro analisado, coeficiente de variação e desvio padrão.

Foi realizada análise de trilha para decompor o coeficiente de correlação em efeitos diretos e indiretos e fazer inferências sobre as relações de causa-efeito entre as variáveis dependentes e as independentes (LOPES; FRANKE, 2009).

A densidade básica foi determinada como atributo dependente e os demais como explicativos, considerando o efeito da colinearidade com uso da constante $\mathrm{k}(0,07)$ no modelo para o atendimento dos critérios relatados por Pereira et al. (2013), onde os valores de inflação da variância são menores que 10 e os números de condições, menor que 100

A análise de fatores foi feita segundo os critérios estabelecidos por Figueiredo Filho; Silva Júnior (2010), com extração dos fatores feita pelo método dos componentes principais e uso da rotação varimax para estabelecimento das cargas fatoriais e facilitar a visualização da relação entre as variáveis não demostradas na análise de trilha. 


\section{Resultados e Discussão}

A densidade básica média obtida para a madeira de teca neste estudo $\left(0,51 \mathrm{~g} \cdot \mathrm{cm}^{-3}\right)$ é coerente com estudos realizados por outros autores (Tabela 1).

Flórez et al. (2014), ao avaliarem a densidade básica em 9 árvores de Tectona grandis com 13 anos de idade, localizadas no noroeste do estado de Minas Gerais, encontraram valor médio de 0,53 g.cm ${ }^{-3}$. Gonçalves et al. (2007), ao estudarem a madeira de teca oriunda do estado de São Paulo e Espírito Santo, com 5 e 12 anos, respectivamente, os autores encontraram valor médio para a densidade básica de 0,40 a 0,47 g. $\mathrm{cm}^{-3}$ para as madeiras oriundas do Espírito Santo e de 0,41 a 0,46 g.cm $\mathrm{cm}^{-3}$ para as madeiras de São Paulo.

A densidade básica da madeira, embora seja de grande importância por estar relacionada ao comportamento físico e mecânico da madeira, varia em função de vários fatores como a idade e variação genética. Segundo Lima et al. (2011) esta diferença pode estar relacionada às condições edafoclimáticas do povoamento e espaçamento entre árvores, sendo que quanto maior for o espaçamento entre árvores, mais longas serão as fibras e mais espessas serão suas paredes celulares, além da menor frequência dos vasos, culminando em maior densidade da madeira.

Conforme classificação de Welling (1994) pode-se observar (Tabela 1) que a madeira estudada apresentou valor de colapso moderado $(2,53 \mathrm{~mm})$ após ser submetida à alta temperatura. Valores moderados de colapso podem ser explicados pela baixa densidade básica da madeira. No caso da teca, mesmo com a secagem forçada a altas temperaturas o baixo volume de lúmem resulta em baixa área colapsada/deformada.

O coeficiente de variação é considerado ótimo até $10 \%$ e regulares entre 10 e $20 \%$. Neste estudo, para a densidade, os valores estão classificados como ótimos. No entanto, para o colapso o valor se aproxima do limite máximo adequado. A oscilação dos valores de coeficiente de variação está em função das propriedades físicas das madeiras, justificada pela heterogeneidade da estrutura química e anatômica.

De acordo com a Tabela 2, os parâmetros físicos estão de acordo com o esperado para a madeira de teca, alta estabilidade dimensional e baixo coeficiente de retração (COSTA et al., 2007), com a retração volumétrica inferior a $12 \%$ e o coeficiente de anisotropia inferior a 2,5.

Observa-se na Tabela 2 que a retração na direção tangencial é, aproximadamente, duas vezes maior que na direção radial para as duas variáveis (retração parcial e total). Esse fato pode ser justificado pela disposição horizontal das células parenquimáticas que constituem os raios que, no plano de corte longitudinal radial, restringem a variação dimensional na direção radial, devido à baixa e, muitas vezes, desprezível retração de cada unidade celular no seu maior eixo.

Valores similares aos obtidos neste estudo foram relatados por Castro; Raigosa (2000) para a Tectona grandis com 17 anos de idade. Já a retração volumétrica foi inferior à obtida por Castro; Raigosa (2000), resultando em menor retração volumétrica da madeira sendo, portanto, mais indicada para indústria moveleira e construção civil. Já o coeficiente anisotrópico foi similar, demostrando a grande estabilidade dimensional para esta espécie, independente da procedência.

Os resultados da análise de trilha demostram a baixa associação entre os parâmetros avaliados e a densidade, em virtude de os efeitos da variável residual $(0,84)$ terem sido superiores ao coeficiente de determinação $(0,30)$ (Tabela 3$)$.

Segundo Nogueira et al. (2012) as variáveis utilizadas no modelo não explicam a variação da densidade básica, sendo que, o baixo coeficiente de determinação verificado, indica a necessidade de inclusão de novos caracteres no diagrama de trilha. 
Tabela 1. Estatística descritiva da densidade básica e do colapso de Tectona grandis.

Table 1. Descriptive statistics of the basic density and the collapse of Tectona grandis.

\begin{tabular}{ccc}
\hline Variável & Densidade $\left(\mathrm{g} . \mathrm{cm}^{-3}\right)$ & Colapso $(\mathrm{mm})$ \\
\hline Mínima & 0,472 & 1,77 \\
Média & 0,510 & 2,53 \\
Máxima & 0,546 & 3,39 \\
$\mathrm{CV}_{\text {exp } \%}$ & 3,70 & 16,87 \\
\hline
\end{tabular}

$\mathrm{CV}_{\text {exp: }}$ coeficiente de variação

Tabela 2. Valores médios para as retrações parciais e totais com seus respectivos coeficientes de variação para a madeira de Tectona grandis.

Table 2. Medium values for partial and total retractions with their respective coefficients of variation for Tectona grandis wood.

\begin{tabular}{|c|c|c|c|c|c|c|c|}
\hline \multirow{2}{*}{ Parâmetro } & \multicolumn{3}{|c|}{ Retração Parcial (\%) } & \multicolumn{3}{|c|}{ Retração Total (\%) } & \multirow{2}{*}{$\mathrm{CA}$} \\
\hline & $\operatorname{Rad}$ & $\mathrm{Tg}$ & Vol & $\operatorname{Rad}$ & $\mathrm{Tg}$ & Vol & \\
\hline Valor médio & 1,24 & 2,11 & 3,6 & 1,75 & 3,49 & 5,23 & 1,99 \\
\hline $\mathrm{CV} \%$ & 30,6 & 32,3 & 32,1 & 29,3 & 30,1 & 32,3 & 31,63 \\
\hline
\end{tabular}

Retração Radial, [Rad], Retração Tangencial [Tg], Retração Volumétrica [Vol], Coeficiente de anisotropia [CA], Coeficiente de Variação [CV].

Tabela 3. Análise de Trilha, com a densidade básica como atributo dependente e os demais como explicativos, em Tectona grandis.

Table 3. Track analysis, with the basic density as dependent attribute and the others as explanatory, in Tectona grandis.

\begin{tabular}{ccccccccccc}
\hline Atributos & R Rad P & R Tg P & R V P & R Rad F & R Tg F & R V F & CA & Colapso & Cor. Db \\
\hline R Rad P & $\mathbf{- 0 , 1 8}$ & 0,02 & 0,56 & 0,12 & $-0,24$ & $-0,20$ & $-0,12$ & $-0,08$ & $-0,13$ \\
R Tg P & $-0,14$ & $\mathbf{0 , 0 3}$ & 0,62 & 0,10 & $-0,22$ & $-0,17$ & $-0,12$ & $-0,12$ & $-0,03$ \\
R V P & $-0,15$ & 0,02 & $\mathbf{0 , 6 5}$ & 0,13 & $-0,32$ & $-0,23$ & $-0,08$ & $-0,08$ & $-0,01$ \\
R Rad F & 0,11 & $-0,01$ & $-0,42$ & $\mathbf{- 0 , 2 0}$ & 0,43 & 0,31 & 0,14 & $-0,04$ & 0,31 \\
R Tg F & 0,08 & $-0,01$ & $-0,39$ & $-0,16$ & $\mathbf{0 , 5 3}$ & 0,32 & $-0,07$ & $-0,09$ & 0,24 \\
R V F & 0,10 & $-0,01$ & $-0,45$ & $-0,18$ & 0,51 & $\mathbf{0 , 3 3}$ & 0,03 & $-0,06$ & 0,28 \\
CA & $-0,05$ & 0,01 & 0,11 & 0,06 & 0,09 & $-0,02$ & $\mathbf{- 0 , 4 3}$ & $-0,08$ & $-0,33$ \\
Colapso & $-0,07$ & 0,02 & 0,25 & $-0,04$ & 0,23 & 0,10 & $-0,17$ & $\mathbf{- 0 , 2 0}$ & 0,10 \\
\hline
\end{tabular}

Umidade relativa (UR), retração radia parciall ( $R$ Rad $P$ ), retração tangencia parcial ( $\mathrm{R}$ tg $\mathrm{P})$, retração volumétrica parcial ( $\mathrm{R}$ Vol P), retração radia parciall $(R$ Rad $T)$, retração tangencia total $(R \operatorname{tg} T)$, retração volumétrica total $(R$ Vol $T)$, coeficiente de anisotropia (CA). Correlação com Densidade básica (cor. DB): significativo a $0,1 \%{ }^{*} \cdot R^{2}=0,30$ e efeito da variável residual = 0,84 .

A relação não significativa entre a densidade básica e as demais propriedades físicas (retrações e colapso) pode ser explicada pela heterogeneidade química e anatômica da madeira e pelo seu processamento. Embora as retrações e, consequentemente, a probabilidade de ocorrência do colapso, estejam de algum modo relacionados à densidade da madeira, outros fatores podem afetar sua retratibilidade, como o tamanho e a geometria das células que a constituem, a velocidade de secagem, o diâmetro do disco e a idade da árvore (GLASS; ZELINKA, 2010; REZENDE et al., 1995).

Para que a avaliação da associação entre caracteres tenha uma estimativa e possa gerar uma interpretação biologicamente apropriada e segura, é de fundamental importância que se teste o grau de colinearidade entre as variáveis independentes. No entanto, quando ocorre inter-relação entre as variáveis independestes estudadas, passa a ser considerado 
como multicolinearidade, e, também deve-se testar o grau em que se manifesta (ISMAEL FILHO et al., 2015).

De acordo com Figueiredo Filho; Silva Júnior (2010) os valores aceitáveis de multicolinearidade estão acima de 0,50 e assim, caso alguma variável apresente valor abaixo desse patamar a mesma deve ser excluída e a análise fatorial deve ser refeita, visando reduzir efeitos adversos da multicolinearidade (Tabela 4).

No presente estudo, seguindo este critério, a anisotropia foi excluída da análise o que demonstra a sua fraca associação com as demais variáveis. $\mathrm{O}$ valor de $\mathrm{K}$ foi encontrado dentro do intervalo $0<\mathrm{k}<1$, sendo que $\mathrm{X}^{\prime} \mathrm{X}$ encontra-se no formato de correlações.

Os valores em negrito em cada componente indicaram os atributos que apresentam maior associação entre si. Para os dois grupos componentes principais, o CP1 explicou 49\% e PC2 93\% da variância acumulada para os dois componentes, respectivamente.

No primeiro componente (PC1), maior relação foi observada entre as retrações totais (radial, tangencial e volumétrico), sendo todos com sinal negativo. Portanto, dentro do componente PC1 houve correlação entre as variáveis, indicando que o aumento do valor de uma das variáveis está relacionado com aumento das demais.

No segundo componente principal, PC2, foram agrupadas as variáveis retrações parciais (radial, tangencial e volumétrico) e o colapso, também apresentando sinal negativo. Apesar de as variáveis terem sido bem mais próximas, para este grupo temos o leve distanciamento da variável retração tangencial. Isso ocorreu porque dentre as retrações, as lineares são as que mais sofrem retrações, podendo então ser influenciada, além da anatomia da madeira, pela sua densidade básica. Portanto, assim como para o PC1, para o PC2 é feita correlação entre as variáveis.

Assim como para a análise de trilha, apenas um eixo linear não é o bastante para inferir significância nos resultados, e por isso os componentes que se agruparam no grupo 1 demonstraram maior associação para as retrações parciais. $\mathrm{O}$ grupo 2 se assemelhou melhor entre as contrações totais e o colapso. Deste modo, o estudo das propriedades físicas e sua relação é importante para dar respaldo na classificação da madeira e indicar diferentes usos.

Tabela 4. Autovalores, variância e variância acumulada (\%) e cargas fatoriais associados aos componentes principais (PC) extraídos a partir de atributos avaliados em Tectona grandis.

Table 4. Eigenvalues, variance and cumulative variance (\%) and factorial loads associated to the main components (PC) extracted from attributes assessed in Tectona grandis.

\begin{tabular}{ccc}
\hline Atributos & PC1 & PC2 \\
\hline R Rad P & 0,41 & $\mathbf{- 0 , 8 2}$ \\
R Tg P & 0,28 & $\mathbf{- 0 , 9 3}$ \\
R V P & 0,49 & $\mathbf{- 0 , 8 5}$ \\
R Rad T & $\mathbf{- 0 , 8 8}$ & 0,30 \\
R Tg T & $\mathbf{- 0 , 9 6}$ & 0,12 \\
R V T & $\mathbf{- 0 , 9 5}$ & 0,27 \\
Colapso & $-0,56$ & $\mathbf{- 0 , 8 0}$ \\
\hline Autovalor & 3,43 & 3,08 \\
Variância & 0,49 & 0,44
\end{tabular}

Retração radia parciall (RT Rad P), retração tangencia parcial (R tg P), retração volumétrica parcial (R Vol P), retração radia parciall ( $R$ Rad T), retração tangencia total ( $\mathrm{R}$ tg $\mathrm{T})$, retração volumétrica total ( $\mathrm{R}$ Vol T) e coeficiente de anisotropia (CA). 


\section{Conclusões}

A madeira de teca apresentou média densidade básica e moderado grau de colapso. As retrações volumétricas, parcial e total, foram baixas, e quando analisadas juntamente com o coeficiente de anisotropia indicam alta estabilidade dimensional da madeira.

A análise de correlação foi significativa, entretanto indicou baixa correlação entre a densidade da madeira e os demais parâmetros avaliados.

A análise dos componentes principais extraídos indicou maior correlação entre as variáveis retrações totais no componente PC1. Para o PC2 a maior correlação foi obtida para as retrações parciais e o colapso.

\section{Referências}

ASSOCIAÇÃO BRASILEIRA DE NORMAS TÉCINAS ABNT. Normas Técnicas NBR 7190: projetos de estrutura de madeira. Rio de Janeiro, 1997. 170p.

ASSOCIAÇÃO BRASILEIRA DE NORMAS TÉCINAS ABNT. Normas Técnicas. NBR 11941: densidade básica da madeira. Rio de Janeiro, 2003 6p.

BRAZ, R. L.; DUARTE A. P. C.; OLIVEIRA, J. T. S.; MOTTA, J. P.; ROSADO, A. M. Curva característica de Secagem da Madeira de Tectona grandis e Acacia mangium ao Ar Livre. Floresta e Ambiente, v. 22, n. 1, p. 117-123. 2015.

CASTRO, F., RAIGOSA, J. Crecimiento y propriedades fisico-mecanicas de la madera de teca (Tectona grandis) de 17 anos de edad em San Joaquin de Abangares, Costa Rica. Agronomia Costarricense, v.24, p. 7-23, 2000.

COSTA, R. B.; RESENDE, M. D. V.; SILVA, V. S. M. Experimentação e seleção no melhoramento genético de

TECA (Tectona grandis L.f.) Floresta e Ambiente, v. 14, p. 76-92, 2007.
FLÓREZ, J. B.; TRUGILHO, P. F.; LIMA, J. T.; GHERARDIHEIN, P.R.; SILVA, J. R. M. Caracterización de la madera joven de Tectona grandis L. f. plantada en Brasil. Madera y Bosques, v. 20, p. 11-20, 2014.

FIGUEIREDO FILHO, D. B.; SILVA JÚNIOR, J. A. Visão além do alcance: uma introdução à análise fatorial. Opinião Pública, v. 16, p. 160-185, 2010.

ISMAEL FILHO, A.; BORGES, P. F.; ARAÚJO, L. S.; PEREIRA, A. R.; LIMA, E. M., SILVA; L. S.; SANTOS JUNIOR, C.V. Influência das variáveis climáticas sobre a evapotranspiração. Gaia Scientia, v. 9, n. 1, p. 62-66, 2015.

GLASS, S. V.; ZELINKA, S. L. Moisture Relations and Physical Properties of Wood. In: Wood handbook-Wood as an engineering material. 508 p. Madison, WI. Chapter 4. 2010.

GONÇALVES, M. P. M.; COFFLER, R.; CARVALHO, A. M.; GARCIA, R. A. Variação radial da densidade básica e comprimento das fibras da madeira de Tectona grandis L. Floresta e Ambiente, v. 14, n. 1, p. 70-75, 2007.

GONÇALVES, F. G.; OLIVEIRA, J. T. S.; DELLA LUCIA, R.M.; NAPPO, M.E.; SARTÓRIO, R.C. Densidade e variação dimensional de um híbrido clonal de Eucalyptus urophylla $\mathrm{x}$ Eucalyptus grandis. Árvore, v. 33, n. 2, p. 277-288, 2009.

INDÚSTRIA BRASILEIRA DE ÁRVORES - IBÁ (2014). Anuário estatístico da IBÁ 2014: ano base 2013. Brasília: IBÁ, 2014. 100 p.

INDÚSTRIA BRASILEIRA DE ÁRVORES - IBÁ (2015). Anuário estatístico da IBÁ 2014: ano base 2014. Brasília: IBÁ, 2014. 100 p.

KLITZKE, R.J. Secagem da madeira. Curitiba: UFPR, 2003.

KLITZKE, R. J.; BATISTA, D. C. Ensaio de taxa de secagem e escore de defeitos para a predição da qualidade de secagem convencional da madeira de Eucalyptus. Scientia Forestalis, v. 38 , n. 85 , p. $97-105,2010$.

LIMA, I. L.; PIMENTEL, M. M.; GARCIA, J. N. Propriedades Mecânicas e Densidade Aparente da Madeira de Tectona grandis Linn. F. (Teca) em Função do Espaçamento e da Posição Radial na Tora. Silva Lusitana, v. 19, p. 221-232, 2011.

LIMA, I. L.; GARCIA, R.; LONGUI, E. L.; FLORSHEIM, S. M. B. Dimensões anatômicas da madeira de Tectona grandis Linn. em função do espaçamento e da posição radial do tronco. Scientia Florestalis, v. 39, n. 89, p. 61-68, 2011. 
LOPES, R. R.; FRANKE, L. B. Análise de trilha dos componentes do rendimento de sementes de trevo-branco. Revista Brasileira de Zootecnia, v.38, p.1865-1869, 2009.

MOTTA, J. P.; OLIVEIRA, J. T. S.; PAES, J. B.; ALVES, R. C.; DAMBROZ, G. B. V. Resistência natural da madeira de Tectona grandis em ensaio de laboratório. Ciência Rural, v. 43, p. 1393-1398. 2013

NOGUEIRA, A. P. O.; SEDIYAMA, T.; SOUSA, L. B.; HAMAWAKI, O. T.; CRUZ, C. D.; PEREIRA, D. G., MATSUO, E. Análise de trilha e correlações entre caracteres em soja cultivada em duas épocas de semeadura. Bioscience Journal, v. 28, p. 877-888, 2012.

PEREIRA, B. F.; VALE, J. C.; CARNEIRO, P. C. S.; FRITSCHE-NETO. R. Relação entre os caracteres determinantes das eficiências no uso de nitrogênio e fósforo em milho. Scientia Forestalis, v. 41, p. 217-225, 2013.

POUBEL, D. S.; GARCIA, R. A.; LATORRACA, J. V. F.; CARVALHO, A. M. Estrutura anatômica e propriedades físicas da madeira de Eucalyptus pellita F. Muell. Floresta e Ambiente, v. 18, p. 117-126, 2011.

SKAAR, C. J. Water in wood. Syracuse: Syracuse University. 1972, 218 p.

SILVEIRA, L. H. C.; REZENDE, A. V.; VALE, A. T. Teor de umidade e densidade básica da madeira em nove espécies comerciais amazônicas. Acta Amazônica, v. 43, p. 179-184, 2013.

WELLING, J European Drying Group- Recommendation. Assessment of drying quality of timber. 1994, 30p. 